\title{
Valeurs implicites dans l'enseignement de la génétique humaine dans les manuels scolaires de cinq pays riverains de la Méditerranée
}

Boujemaa Agorram, Silvia Caravita, Jérémy Castéra, Pierre Clément, Férida Khammar et Sabah Selmaoui

\section{(2) OpenEdition}

\section{Journals}

Édition électronique

URL : http://journals.openedition.org/trema/2638

DOI : $10.4000 /$ trema.2638

ISSN : 2107-0997

Éditeur

Faculté d'Éducation de l'université de Montpellier

Édition imprimée

Date de publication : 1 décembre 2011

Pagination : $8-20$

ISSN : 1167-315X

Référence électronique

Boujemaa Agorram, Silvia Caravita, Jérémy Castéra, Pierre Clément, Férida Khammar et Sabah Selmaoui, «Valeurs implicites dans l'enseignement de la génétique humaine dans les manuels scolaires de cinq pays riverains de la Méditerranée », Tréma [En ligne], 35 - 36 | 2011, mis en ligne le 01 décembre 2013, consulté le 19 avril 2019. URL : http://journals.openedition.org/trema/2638; DOI : 10.4000/trema.2638

Ce document a été généré automatiquement le 19 avril 2019

Trema 
Valeurs implicites dans l'enseignement de la génétique humaine dans les manuels scolaires de cinq pays riverains de la Méditerranée

Boujemaa Agorram, Silvia Caravita, Jérémy Castéra, Pierre Clément, Férida Khammar et Sabah Selmaoui

\section{Introduction}


1 La génétique humaine a connu ces dernières années une vraie révolution scientifique (au sens de KUHN, 1970) ${ }^{1}$. Alors qu'elle a longtemps été marquée par le débat "inné" ou "acquis", auquel a succédé une formulation plus consensuelle, sur un modèle additif, inné et acquis (la part de l'un et de l'autre dans le déterminisme des comportements et performances humaines), il est devenu scientifiquement évident que les termes de ce débat étaient dépassés, puisque inné et acquis sont obligatoirement en interaction, les deux étant nécessaires autant l'un que l'autre ( STEWART 1993, JACQUARD et KAHN, 2001). La volonté de réduire les comportements ou la personnalité humaine à un déterminisme génétique (la recherche du gène de

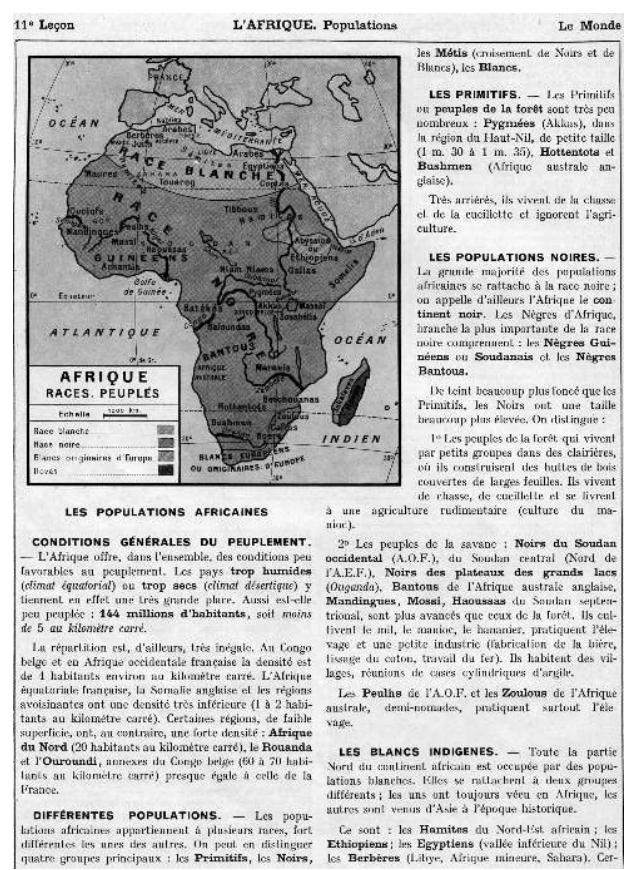
l'intelligence, de l'agressivité, de la fidélité ou de l'homosexualité) est dès lors apparue comme idéologique, au sens proposé par le philosophe de la biologie G. CANGUILHEM $(1981)^{2}$. Plusieurs travaux ont dénoncé cette idéologie réductionniste (citons, entre autres : CLÉMENT et al., 1981; LEWONTIN et al., 1984 ; RUMELHARD, 1986 ; STEWART, 1993 ; ATLAN et BOUSQUET, 1994 ; CLÉMENT et FORISSIER, 2001 ; KUPIEC et SONIGO, 2001 ; JACQUARD et KAHN, 2001 ; LEWONTIN, 2003).

2 Ces travaux ont marqué la fin du tout-génétique pour reprendre le titre de l'ouvrage d' ATLAN (1999) et l'avènement d'un nouveau paradigme, l'épigénétique (MORANGE, 2005a, 2005b). Au sens strict, ce terme désigne les interactions entre le génome et son environnement direct, interaction dont l'importance est devenue évidente à l'issue du séquençage du génome humain (2001) : 25000 gènes seulement alors que les estimations antérieures en attendaient 100000 à 150000 selon l'hypothèse que tout ce qui distingue l'être humain des autres animaux aurait des supports génétiques. Au sens large, l'épigénétique désigne les mécanismes par lesquels le génotype ${ }^{3}$ engendre le phénotype ${ }^{4}$ : des processus non génétiques mais en interaction avec lui (étymologiquement, épigénétique, au dessus des gènes). La formation des synapses, qui dépend des contacts que l'organisme établit avec son environnement, fait partie des phénomènes épigénétiques (épigenèse cérébrale). On sait aujourd'hui que toutes nos performances se construisent par nos apprentissages moteurs et intellectuels, mémorisés dans nos réseaux neuronaux (CHANGEUX, 1983, 2002 ; EDELMAN, 1987). Ce nouveau paradigme ne dit pas que rien, chez les humains, ne serait inné ou au contraire que tout le serait. Il signifie que l'inné ne peut pas être séparé de l'acquis, puisqu'il est en interaction avec lui, et que l'acquis ne peut être séparé de l'inné (du génotype humain qui permet l'épigenèse cérébrale, par exemple).

Notre question est donc de savoir dans quelle mesure ce nouveau paradigme a ou non été intégré dans les enseignements scolaires de génétique, en nous focalisant sur les manuels scolaires de biologie. Nous nous proposons d'analyser ici les conceptions identifiables dans les manuels scolaires en tant qu'interactions entre connaissances scientifiques (Knowledge) et valeurs (V), dans le cadre du modèle KVP proposé par Pierre CLÉMENT 
(2004, 2006). L'enjeu de cette interaction KV concerne les pratiques sociales (P), qui seraient plus fatalistes si l'idéologie innée s'avère encore très prégnante, alors qu'elles seraient plus constructivistes si le contenu de l'épigénétique (à défaut du terme luimême) est enseigné.

Nous privilégierons quelques indicateurs de la persistance éventuelle de l'innéisme (la fréquence des termes "programme génétique") dans les chapitres de génétique humaine, après avoir recensé l'importance de ces chapitres au cours de la scolarité. En outre, nous prendrons en compte d'éventuels indicateurs sexistes ou racistes, même si la question du genre dans les systèmes éducatifs est bien étudiée (voir par exemple ARNOT et MAC AN GHAILL, 2006; WINTER, 2006; SORENSEN, 2007; MARGUERITE, 2008), y compris dans les manuels de mathématiques de divers pays africains francophones (BRUGEILLES et CROMER, 2008).

Notre recherche s'intègre dans le cadre du projet de recherche Biohead-Citizen ${ }^{5}$ (Biology, Health and Environmental Education for better Citizenship), qui porte sur 6 thèmes (dont la génétique humaine) dans 19 pays. Seuls les pays méditerranéens de ce projet sont concernés par le présent travail, avec l'hypothèse de plus de traces d'innéisme et de sexisme dans les pays du Sud de la Méditerranée que dans ceux du Nord. En effet, des différences ont été observées à propos des conceptions d'enseignants (primaire, secondaire biologie et secondaire lettres), qui varient fortement selon les pays sur des questions vives telles que la justification génétique d'une prédisposition des femmes à faire le ménage ou de la supériorité de certains groupes ethniques sur d'autres. En France et en Italie, les enseignants ne justifiaient pas ces différences par des arguments génétiques, à l'inverse des enseignants de trois pays du Maghreb (Maroc, Algérie et Tunisie), qui, par ailleurs, diffèrent entre eux (CASTÉRA et al., 2007 ; CLÉMENT et al., 2008).

\section{Corpus étudié et méthode d'analyse}

Pour connaître l'importance accordée au thème de la génétique dans les programmes, nous avons compté le nombre de niveaux où la génétique est enseignée dans les 5 pays méditerranéens choisis et le nombre de pages allouées au thème de génétique générale et de génétique humaine dans les différents manuels.

\section{1. Corpus étudié}

4 Nous avons analysé 28 manuels (cf. corpus en bibliographie) : France (11 livres), Tunisie (3), Maroc (4), Italie (7), Algérie (4). La différence entre le nombre des manuels scolaires est due à deux paramètres : dans certains pays, la génétique humaine est enseignée à deux niveaux alors que dans les autres, ce thème est enseigné dans plus de deux niveaux; dans certains pays, il n'existe qu'un seul éditeur officiel (le ministère ou son représentant), alors que dans d'autres, il existe plusieurs éditeurs privés.

\section{2. Méthode d'analyse}

5 Nous avons utilisé la grille spécifique au thème génétique, collectivement construite et améliorée par les différentes équipes du projet européen de recherche Biohead-Citizen ( CARVALHO et al., 2004, 2006). Nous avons axé le présent travail sur les points suivants de la grille : 
1 - Nombre de pages allouées au thème de génétique générale et de génétique humaine.

2 - Occurrence des expressions "programme génétique » et "information génétique ». Ces termes sont en effet significatifs; ils indiquent si les manuels ont tendance à être ou ne pas être en rupture avec l'ère jusque-là prédominante du "tout génétique" selon laquelle nous serions largement programmés par nos gènes (idéologie fataliste). Nous signalons les occurrences des mots "programme » et «information» lorsque ces mots sont associés (explicitement ou implicitement) avec "génétique »: "programme génétique » et « information génétique ». Cette méthode des termes-pivots (HARRIS, 1952) a été notamment utilisée par JACOBI (1987), ABROUGUI (1997), CASTÉRA et al. (2008) pour analyser certains contenus de manuels de biologie.

En parallèle, nous avons vérifié comment les manuels traitent ou non de l'interaction du génome avec l'environnement pour engendrer le phénotype ou s'ils présentent des conceptions réductrices du type : un gène détermine un phénotype.

3 - Comptage des personnages selon leur sexe (masculin-féminin) dans les images, pour y déceler d'éventuelles conceptions sexistes.

4 - Présence ou non d'une diversité ethnique dans les images et les photos, pour y déceler d'éventuelles conceptions ethnocentrées (voire racistes).

\section{Résultats et discussion}

\section{1. Place de la génétique dans le cursus d'études}

7 Les 5 pays diffèrent quant aux niveaux d'enseignement de la génétique. 4 niveaux sont concernés en France et en Algérie (mais pour l'Algérie, un des programmes traite seulement le thème de l'amélioration du rendement en agriculture pour les 15-16 ans), 3 niveaux pour la Tunisie et l'Italie (si l'on inclut, pour l'Italie, la Biologie moléculaire programmée pour les 18-19 ans) et seulement 2 niveaux pour le Maroc. Pour l'Italie, la génétique au sens strict est enseignée 2 années entre $11 / 13$ ans et les $14 / 17$ ans : les enseignants ont le libre choix de la programmer dans 2 niveaux parmi les 5. Pour l'Italie et le Maroc, la génétique est enseignée dès le collège.

Le nombre de pages est très variable selon les pays et selon les manuels d'un même pays quand il en existe plusieurs. Le nombre de pages réservées à la génétique est plus important en France et au Maroc, suivis par la Tunisie et l'Algérie. Les manuels de l'Italie sont en retrait et réservent moins de pages à ce thème. Mais si on se réfère seulement au thème de la génétique humaine, la France, la Tunisie et l'Italie y consacrent plus de pages que l'Algérie et le Maroc.

\section{2. Occurrences de " programme » et « information » génétique}

8 Le terme "programme génétique " est plus présent dans les manuels français et marocains (anciens manuels) que dans ceux de l'Algérie, de l'Italie et de la Tunisie où le terme programme est très rare, voire totalement absent (manuels tunisiens). Le terme « information génétique » est plus utilisé dans les manuels français, italiens et tunisiens que par ceux du Maroc et de l'Algérie (tableaux 1 et 2). 
Tableau 1 : Occurrences du «programme génétique» dans les manuels des 5 pays méditerranéens

\begin{tabular}{|c|c|c|c|c|c|c|c|c|}
\hline \multicolumn{9}{|c|}{ Age des élèves } \\
\hline Pays & $11-12$ & $12-13$ & $13-14$ & $14-15$ & $15-16$ & $16-17$ & $17-18$ & $18-19$ \\
\hline France & & & & $66 / 7 *$ & $18 / 49^{*}$ & $1 / 2^{*}$ & $0 / 0^{*}$ & \\
\hline Italie & \multicolumn{2}{|c|}{$0 / 0 / 0^{*}$} & & \multicolumn{3}{|c|}{$2 / 0 / 0 / 0^{*}$} & & \\
\hline Maroc & & & $-23^{*}$ & & & & $28-5 / 1^{* *}$ & \\
\hline
\end{tabular}

Note de lecture :

" Le " / " sépare les manuels des différents éditeurs (en France, en Italie et au Maroc)

** la génétique n'était pas enseignée au Maroc pour le niveau 13-14 ans avant la réforme du système éducatif de 1999 ; pour les 17-18 ans, le tiret sépare les chiffres des manuels de l'avant et l'après- réforme.

Tableau 2 : Occurrences de «information génétique»dans les manuels des 5 pays méditerranéens

\begin{tabular}{|c|c|c|c|c|c|c|c|c|}
\hline \multicolumn{7}{|c|}{ Age des élèves } \\
\hline Pays & $11-12$ & $12-13$ & $13-14$ & $14-15$ & $15-16$ & $16-17$ & $17-18$ & $18-19$ \\
\hline France & & & & $9 / 73^{*}$ & $81 / 59^{*}$ & $27 / 28^{*}$ & $15 / 1^{*}$ & \\
\hline Italie & \multicolumn{2}{|c|}{ Souvent utilisé } & & \multicolumn{3}{|c|}{ Souvent utilisé } & & \\
\hline Maroc & & & $-0^{* *}$ & & & & $10-22 / 39^{* *}$ & \\
\hline Algérie & & & & & 7 & 6 & 0 & \\
\hline Tunisie & & & & 7 & & & 3 & 30 \\
\hline
\end{tabular}

Note de lecture:

* Le " / " sépare les manuels des différents éditeurs (en France, en Italie et au Maroc)

** la génétique n'était pas enseignée au Maroc pour le niveau 13-14 ans avant la réforme du système éducatif de 1999 ; pour les 17-18 ans, le tiret sépare les chiffres des manuels de l'avant et l'après- réforme.

On note une grande disparité entre les manuels français qui l'utilisent de moins en moins du collège au lycée. Les manuels français publiés en 1995 utilisaient beaucoup plus le terme de programme, témoignant d'un héréditarisme ${ }^{6}$ implicite (ABROUGUI, 1997, CLÉMENT et FORISSIER, 2001), mais avec des différences substantielles d'un éditeur à un autre ( FORISSIER et CLÉMENT, 2003).

Pour la France, nous avons comparé les manuels scolaires de trois éditeurs différents. Chez Nathan, les occurrences de « programme génétique » diminuent au cours du cursus, du collège au lycée. Chez Hatier, le déterminisme génétique dans le manuel scolaire du premier niveau est dominé par la notion d'"information génétique", le deuxième niveau par le "programme génétique », puis de nouveau par «l'information génétique». La comparaison avec les manuels français publiés en 1990 montre qu'aujourd'hui, le terme de "programme génétique» tend à être remplacé par la notion d'«information génétique ». Néanmoins l'expression "programme génétique » n'a pas disparu, ce qui suggère l'influence d'autres paramètres.

Le premier de ces paramètres est qu'au niveau du collège, le projet pédagogique initie les élèves les plus jeunes à la génétique en utilisant le terme " programme génétique ", parce que ce serait un message simple et important: le génotype détermine le phénotype. Or cette simplification initiale est dangereuse, car les premières notions enseignées sont les 
plus mémorisées par les élèves et plus résistantes au changement et elles sont difficilement remplacées par des concepts scientifiques (CLÉMENT et al., 2003). Il est aussi possible que la difficulté à supprimer totalement la notion de " programme génétique » découle du fait qu'elle est au cœur des programmes précédents: la notion, avec son corollaire idéologique implicite, est encore présente aussi bien chez les auteurs que les éditeurs et donc dans les manuels.

L'analyse montre encore que les manuels français traitent du rôle de l'environnement dans l'expression du phénotype au niveau du collège (Génétique et environnement: niveau d'âge 14-15 ans) et du lycée (Du génotype au phénotype, relations avec l'environnement; Complexité des relations entre gènes, phénotype et environnement: niveau d'âge 16-17 ans).

11 Les nouveaux manuels du Maroc utilisent nettement moins que l'ancien manuel les termes "programme génétique", qui ont été remplacés en partie par ceux d'«information génétique ». Cette substitution semble consciente et réfléchie par les auteurs du fait de l'impact des résultats de recherches en didactique concernant ce point. En effet, ces auteurs, pour la plupart inspecteurs d'enseignement, ont eu une formation en didactique de la biologie. Cette hypothèse mériterait cependant d'être vérifiée, d'autant qu'il n'y a pas un abandon total des termes «programme génétique » dans ces manuels.

Ces manuels marocains et ceux d'Algérie semblent donc évoluer de la même manière que les manuels français : diminution des occurrences de "programme génétique » et donc affaiblissement d'un héréditarisme implicite, même si cette tendance est relativement tardive.

12 En revanche, les manuels tunisiens utilisent seulement le concept d'«information génétique » aux trois niveaux où la génétique humaine est enseignée. Cela signifie que, pour les chapitres traitant de la génétique humaine, ils ont adopté très rapidement de nouveaux contenus scientifiques, avec un changement plus rapide et plus radical qu'en France, au Maroc et en Algérie. Ce changement rapide est un indicateur de l'influence croissante des chercheurs tunisiens en didactique de la biologie (formation des inspecteurs, participation aux nouveaux programmes). Cependant, l'absence de la notion de « programme génétique » ne signifie pas pour autant une disparition totale des idées innéistes dans les manuels scolaires tunisiens (CLÉMENT, MOUELHI, et ABRoUGUI, 2006), ni dans les conceptions des enseignants tunisiens (коснКAR, 2007). D'ailleurs, l'absence, dans les manuels des trois pays maghrébins, de toute référence au rôle de l'environnement (et de son interaction avec le génome) dans l'expression du phénotype est un indicateur de la persistance de conceptions héréditaristes implicites.

13 En Italie, l'expression « programme génétique » n'est présente, de façon précise, que deux fois et seulement dans un seul manuel (возснетті, 2004). Les manuels italiens utilisent souvent des termes comme "patrimoine héréditaire » et "patrimoine génétique » ( LEOPARDI, 2004 ; MILLER, 2000 ; COLOMBI, 2006 ; PISERI, 2001). Ces expressions sont moins marquées que celle de "programme génétique " et véhiculent des valeurs implicites moins déterministes. L'ensemble de ces résultats suggère la volonté des auteurs d'être prudents en traitant des thèmes pouvant comporter implicitement une idéologie héréditariste. Le passé de l'Italie au cours de la Seconde Guerre mondiale a certainement eu une influence sur la façon, parfois assez maladroite, dont la génétique humaine est enseignée aujourd'hui. Par exemple, dans le manuel de MILLER (2000), on recourt à la métaphore du grand livre de la vie: «Les informations nécessaires à l'expression des 
caractères d'un individu sont contenues dans les gènes, qui sont comme des livres, les gènes sont classés sur les chromosomes, qui sont comme des étagères » (p.87). Selon cette métaphore du «livre de la vie», il suffirait de connaître l'alphabet et la syntaxe génétiques pour parvenir à l'essence de l'être humain. Mais c'est aussi la métaphore biblique du verbe au commencement de tout (début de l'évangile de Jean). Dans le manuel de PISERI (2001), une allusion aux processus épigénétiques peut néanmoins être relevée : "Chaque organisme est souvent le résultat d'une interaction complexe entre les gènes et l'environnement» (p.36). Mais le même manuel utilise aussi une autre expression, qui rappelle la métaphore du «livre de la vie »: «...Chaque cellule est capable de s'autoconstruire elle-même et de fonctionner, car elle contient un projet très précis (ADN) et les structures qui lui permettent de l'interpréter et de l'exprimer»(p.4). L'expression « projet précis » suggère que tout est écrit, en négligeant que la différenciation cellulaire est également le résultat des interactions entre les cellules dans un organisme. Donc, même si les termes "programme génétique » sont quasi absents des manuels scolaires italiens, d'autres expressions montrent la persistance d'un innéisme implicite (projet, projet précis, projet génétique, instructions héréditaires).

\section{3. Diversité ethnique et représentations sexuées}

14 En général, les manuels des 5 pays intègrent des personnages de différentes « ethnies» avec une prépondérance de l'ethnie du pays, ce qui est à l'image des diversités existant dans ces pays (tableau 3).

Tableau 3 : Nombre de personnages représentant une diversité ethnique dans les images des manuels du chapitre Génétique Humaine.

\begin{tabular}{|c|c|c|c|c|c|c|c|c|}
\hline \multicolumn{7}{|c|}{ Age des élèves (en années) } \\
\hline Pays & $11-12$ & $12-13$ & $13-14$ & $14-15$ & $15-16$ & $16-17$ & $17-18$ & $18-19$ \\
\hline France & & & & $12 / 25-6 / 24$ & $0 / 7$ & $4 / 9$ & $1 / 11$ & \\
\hline Italie & $3 / 21^{*}$ & & \multicolumn{2}{|c|}{$5 / 14-3 / 9^{* *}$} & & \\
\hline Maroc & & & $2 / 10$ & & & & $10-22 / 39^{* *}$ & \\
\hline Algérie & & & & $2 / 6$ & & $\mathbf{0}$ & & $\mathbf{0}$ \\
\hline Tunisie & & & & $9 / 7$ & & & 0 & $2 / 2$ \\
\hline
\end{tabular}

Note de lecture :

* Les premiers chiffres indiquent les diversités ethniques (noir, indien, amérindien, eskimo...); les seconds chiffres séparés par un " / " les personnages de couleur peau blanche

** Le tiret sépare les manuels de différents éditeurs

15 Au niveau de la représentation des deux sexes dans l'iconographie des manuels, en France, le sexe féminin soit plus représenté (à l'exception d'un manuel du niveau 17-18 ans) alors qu'au Maroc c'est le sexe masculin, mais il faut souligner que, dans l'ensemble, les différences ne sont pas significatives. Pour l'Algérie et la Tunisie et dans une moindre mesure l'Italie, le nombre de personnages féminins est égal à celui des masculins.

On ne peut pas en conclure pour autant à l'absence de discrimination sexuée dans ces manuels car le comptage des personnes présentes dans les images n'est pas suffisante pour identifier une éventuelle discrimination sexuée. Il aurait fallu analyser aussi les rôles sociaux sexués des différents personnages, aussi bien dans les images que dans les 
textes, pour saisir les mécanismes d'élaboration des représentations du « masculin » et du " féminin » et dévoiler le système de genre d'un manuel. D'autres travaux, qui ont porté sur les manuels de mathématiques de quelques pays africains dont la Tunisie, ont montré ainsi la persistance de représentations sexuées porteuses d'inégalités (BRUGEILLES et CROMER, 2008).

Pour le Maroc (c'est aussi le cas de l'Algérie et de la Tunisie), la production des nouveaux manuels se base sur un cahier de charges qui stipule la nécessité de s'abstenir de toute discrimination basée sur le sexe, la religion ou l'appartenance à une race ou une communauté (en incluant des photos d'autres ethnies). La validation par une commission d'experts de la conformité aux exigences du cahier des charges doit néanmoins pouvoir s'appuyer sur des critères précis.

\section{Conclusion}

L'analyse comparative révèle une certaine survivance des conceptions innéistes et héréditaristes dans le traitement didactique du thème de la génétique dans les manuels des 5 pays retenus, mais de façon différente selon les cas. Si on se réfère à l'introduction du nouveau paradigme de l'épigénétique ou de son contenu, avec des références explicites à l'interaction entre génome et environnement dans l'expression du phénotype, les pays du Maghreb, où cette introduction reste encore timide, s'opposent aux pays du nord de la Méditerranée. En revanche, si on se réfère à l'abandon par les manuels du syntagme "programme génétique", ces mêmes pays de la rive sud de la Méditerranée, plus particulièrement la Tunisie, sont souvent en avance par rapport à ceux de la rive nord. Cet abandon progressif des termes "programme génétique" dans les manuels des pays maghrébins semble dû à la diffusion des recherches en didactique concernant cette question (par exemple la thèse d'ABRoUGUI, 1997). Les auteurs de ces manuels sont, pour la plupart, des enseignants et des inspecteurs formés en didactique, très engagés dans la quête de nouveautés dans la didactique de la biologie en particulier. Certes, ces auteurs s'inspirent essentiellement des manuels français, avec un certain décalage, mais sur cette question précise (occurrence de "programme génétique »), ils ont devancé les manuels français où ce syntagme reste très présent au collège, ce qui traduit le faible impact des recherches en didactique effectuées depuis plus d'une dizaine d'années.

Notre étude montre aussi l'impact positif des cahiers de charges nationaux sur l'écriture des manuels, mais également leurs limites concernant des contenus implicites de ces manuels et surtout au niveau des enseignants eux-mêmes.

Ainsi, les conceptions plus héréditaristes et sexistes des enseignants des pays maghrébins par rapport à celles de leurs homologues français et italiens (CASTÉRA et al., 2007 ; CLÉMENT et al., 2008) correspondent à des conceptions scientifiques dépassées ou à des stéréotypes encore tenaces véhiculés par certaines franges de la société et par certains médias. Ces conceptions et ces stéréotypes sont toutefois contrecarrés par les progrès scientifiques et par la culture d'égalité promue par les réformes sociales entreprises dans ces pays maghrébins depuis quelques années, dont nous avons retrouvé la marque dans les manuels scolaires analysés.

Le manuel scolaire reste « un instrument de premier choix pour introduire un processus de changement social, qui permet à tout un chacun de jouir totalement de ses droits et de 
ne subir aucune forme de discrimination selon son sexe ou ses caractéristiques sociales quelles qu'elles soient » (BRUGEILLES et CROMER 2008).

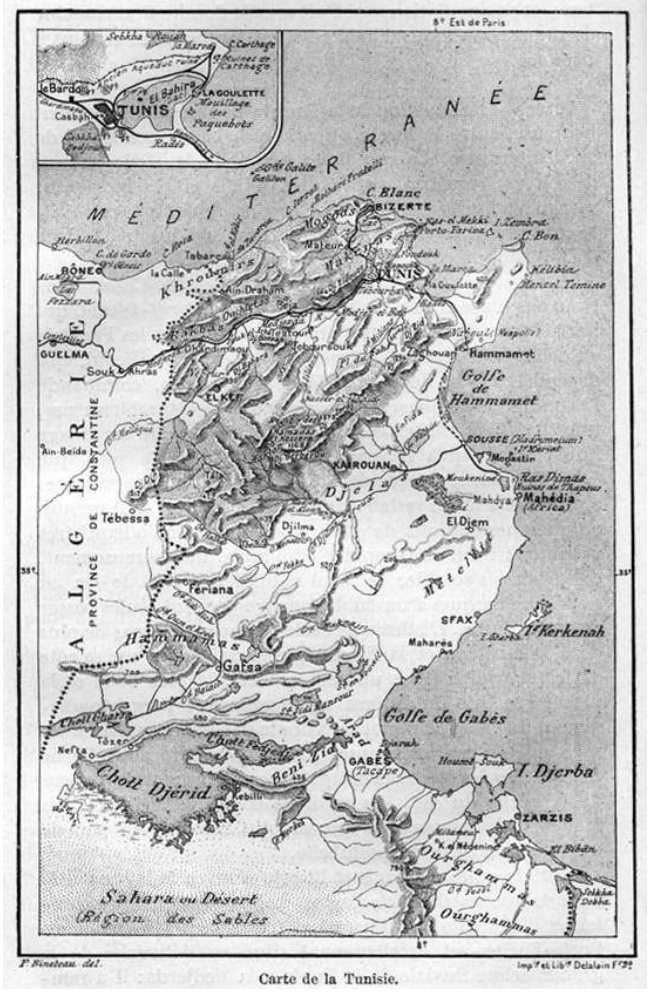

La France, ses colonies et Protectorats, A. GASQUET. Paris : Imprimerie et librairie classiques DELALAIN Frères, 1892, p. 370. Source : CEDRHE.

\section{BIBLIOGRAPHIE}

ABROUGUI M., La génétique humaine dans l'enseignement secondaire en France et en Tunisie. Approche didactique. Thèse de doctorat, Université Lyon 1, 1997

ARNOT M. et MAC AN GHAILL, M., The Routledge Falmer Reader in Gender and Education. London et N.Y. : ROULEDGE, TAYLOR et FRANCIS GROUP, 2006

ATLAN H., La fin du "tout génétique". Paris: INRA, 1999

ATLAN H., et BOUSQUET C. Questions de vie. Entre le savoir et l'opinion. Paris : Seuil, 1994

BRUGEILLES C. et CROMER S., Comment promouvoir l'égalité entre les sexes par les manuels scolaires? Guide méthodologique à l'attention des acteurs et actrices de la chaîne du manuel scolaire. Paris : UNESCO, 2008

CANGULHEM G., Idéologie et rationalité dans l'histoire des sciences de la vie, 2e éd. Paris : Librairie philosophique J. VRIN, 1981

CASTÉRA J. et CLÉMENT P., A gender effect related to teachers' conceptions of biological gender differences : A survey in 14 countries. ERIDOB proceedings (European researchers in didactics of biology), University of Utrecht, 16th -20th September, Sous presse, 2008

CASTÉRA J., MUNOZ F. et CLÉMENT P., Les conceptions d'enseignants du primaire et du secondaire sur le déterminisme biologique de la personnalité humaine dans 12 pays d'Europe, d'Afrique et $\mathrm{du}$ Moyen Orient. Actes du Congrès international, AREF (Actualité de la Recherche en Education et en 
Formation), Strasbourg, 28-31 août, 2007, 12 pages.

Disponible sur internet : http://www.congresintaref.org/actes_pdf/

AREF2007_Jeremy_CASTERA_298.pdf (consulté le 11 septembre 2009)

CHANGEUX J.P., L'homme neuronal. Paris : FAYARD, 1983

CHANGEUX J.P., L’homme de vérité. Paris : Odile JACOB, 2002

CLÉMENT P., Science et idéologie : exemples en didactique et épistémologie de la biologie. Actes du Colloque sciences, médias et société. ENS-LSH, 2004, p.53-69.

http://sciences-medias.ens-lsh.fr

CLÉMENT P., Didactic transposition and the KVP Model: Conceptions as interactions between scientific knowledge, values and social practices. Proceedings summer school ESERA, IEC, Univ. Minho (Portugal), 2006, p. 9-18

CLÉMENT P., BLAES N., BlAineAu S., DeBARd E., JOURDAN F., LuCIANi A., Biologie et société : le matin des biologistes? Paris : Raison présente, 1981

CLÉMENT P. et FORISSIER T., L'identité biologique n'est pas que génétique : un défi pour un enseignement citoyen. Communication au symposium BioEd 2000 : The challenge of the next century, Paris, 15-18 May 2000, 2001

Disponible sur internet : www.iubs.org/cbe/pdf/clement.pdf (consulté le 11 septembre 2009)

ClÉMENT P., LAURENT C., CASTÉRA J. et QUesSADA M.-P., Conceptions d'enseignants et futurs enseignants français et de six pays de la francophonie sur quelques questions vives de biologie (Algérie, Burkina Faso, France, Liban, Maroc, Sénégal, Tunisie). In KHALIL, I., Enjeux dans la rénovation de l'éducation à l'environnement et à la biologie. Alexandrie : presses Université Senghor, sous presse, 2008, 28 pages

EDELMAN G., Neural Darwinism : the theory of neuronal group selection. New York : Basic Books, 1987

HARRIS Z, S., Discourse analysis, Language, 28 (traduction française 1969, dans Langages, 13), 1952

JACOBI D., Textes et images de la vulgarisation scientifique. Berne : Peter LANG, 1987

JACQUARD A. et KAHN A., L'avenir n'est pas écrit. Paris : BAYARD, 2001

KOCHAR M., Les détérminismes biologiques: Analyse des conceptions et des changements conceptuels consécutifs à un enseignement sur l'épigenèse cérébrale chez des enseignants et des apprenants tunisiens. Thèse de doctorat, Université Lyon 1, 2007

KUHN T., La structure des révolutions scientifiques. Paris : FLAMMARION, (Traduction française en 1983 de la seconde édition de 1970), 1970

KUPIEC J.J. et SONIGO P., Ni dieu ni gène. Pour une autre théorie de l'hérédité; Col Science ouverte ; Éditions du Seuil, 2001

LEWONTIN R. C., La triple hélice. Les gènes, l'organisme, l'environnement. Paris : Le Seuil, 2003

LEWONTIN R. C., ROSE S., et KAMIN L. J., Not in our genes: Biology, ideology and human nature. New York : Pantheon, 1984

MARGUERITTE H., Genre et éducation. Dossier d'actualité de L'INRP, nº37 (septembre), 2008 Disponible sur : http://www.inrp.fr/vst/LettreVST/37_septembre2008.htm

MORANGE M., L'épigénétique : un domaine aux multiples facettes. Médecine/Science, n²1, 2005 a, p. 339 
Morange M., Quelle place pour l'épigénétique ? Médecine/Science, n²1, 2005 b, p. 367-369

RUMELHARD G., Les représentations de la génétique. Berne : Peter LANG, 1986

SORENSEN H., Gender inclusive Science education? In CORRIGAN, D., DILLON J. et Gunstone R., (Eds.), The Re-Emergence of Values in Science Education, 2007, p. 249-267 Rotterdamm : Sense Publishers STEWART J., Au delà de l'inné et de l'acquis. Intellectica, 16, 1993, p. 151-174

WINTER B., Religion, culture and women's human rights: Some general political and theoretical considerations. Women's Studies International Forum, 29, 2006, p.381-393

\section{Corpus de manuels}

\section{Algérie}

BOUCHLAGHEM A. et al., Science de la nature et de la vie ; 4e année moyenne ; Éditions ONPS, (14-15 ans), 2006

BOULEOUDINET et al., Sciences de la Vie et de la Terre : TC science et technologie ; 1re année secondaire ; Éditions ONPS, (15-16 ans), 2008

BOUZEKARIA N. et al., Science de la nature et de la vie - classes sciences expérimentales et mathématiques ; 2e année secondaire, Éditions ONPS, (16-17 ans), 2006 BOUCHLAGHEM A. et al., Science de la nature et de la vie - classes sciences expérimentales - 3e année secondaire, Éditions ONPS, (17-18 ans), 2007

\section{France}

AUDEBERT M. et al., Sciences de la vie et de la terre, (16-17 ans). NATHAN, 2004

BAL A. et al., Sciences de la vie et de la terre, (14-15 ans). NATHAN, 2003

BAUDE D. et al., Sciences de la vie et de la terre (littéraire), (16-17 ans). BORDAS, 2004

BAUDE D. et al., Sciences de la vie et de la terre (économique et sociale), (16-17 ans). BORDAS, 2004 BEAUJARD P. et al., Sciences de la vie et de la terre, (15-16 ans). HATIER, 2003 BEAUJARD P. et al., Sciences de la vie et de la terre (scientifique), (16-17 ans). HATIER, 2001 BEAUJARD P. et al, Sciences de la vie et de la terre (scientifique), (17-18 ans). HATIER, 2005 BEAUX J-F. et al, Sciences de la vie et de la terre (scientifique), (17-18 ans). NATHAN, 2002 BEAUX J-F. et al., Sciences de la vie et de la terre (spécialité SVT), (17-18 ans). NATHAN, 2002 BEAUX J-F. et al., Sciences de la vie et de la terre, (15-16 ans). NATHAN, 2000 BRIDIER C. et al., Sciences de la vie et de la terre, (14-15 ans). HATIER, 2004

\section{Maroc}

BERRI M. et al., Al mofid Sciences de la Vie et de la Terre, (13-14 ans). Dar attakafa Casablanca, 2004 
Auteurs non cités, Sciences naturelles. 3e année sciences expérimentales, (17-18 ans). Afriquia Achark.(programme d'avant réforme ), 2005

TACKHALWICHT M. et al., Aljadid des sciences de la vie et de la terre, (17-18 ans). Dar anachr Casablanca, 2008

LAMAAMAR A. et al., Firihab Sciences de la vie et de la terre,(17-18 ans). Adar Aalamia lilkitab Casablanca, 2007

\section{Italie}

BosCHETtI M. et al., Nuovo ecosistema terra Vol SB 1 Gli esseri viventi - La cellula - La genetica, (14-17 ans). Minerva Italica, 2004

CoLOMBi et al., Sperimentare scienze, (11-13 ans). Edizioni Il Capitello, 2006

GAINOTTI A. et al., Biologia - Diversità e unità dei viventi Mod A-D, (14-17 ans). ZANICHELLI, 2004

LEOPARDI L. et al., Nuovo LS - Il libro delle scienze, (11-13 ans). Garzanti scuola, 2004

MILLER K. R., et al., Il mondo della natura. Vol. A-F : Il punto di vistadella Biologia, (14-17 ans). Edizioni scolastiche - Bruno MONDADORI, 2000

PISERI A. et al., VIVERE. Percorsi modulari di Biologia. Vol. D - Ereditarietà e riproduzione, (14-17 ans). LOESCHER, 2001

FLACCAVENTO G. et al., La materia e la natura - L'uomo, (11-13 ans). Fabbri Editori, 2005

\section{Tunisie}

ABROUG R. et al., Sciences naturelles 3e Année de l'Enseignement secondaire section sciences expérimentales, (17-18 ans). Centre national pédagogique. Tunis, 2004

CHADLI H. et al., Sciences naturelles 4e Année de l'Enseignement secondaire section sciences expérimentales, (18-19 ans). Centre national pédagogique. Tunis, 2005

YACOUB T. et al., Sciences naturelles 9e Année de l'Enseignement de base, (14-15 ans). Centre national pédagogique. Tunis, 2005

\section{NOTES}

1. Selon Kuhn, la science progresse de manière fondamentalement discontinue, c'est-à-dire non par accumulations mais par ruptures. Ces ruptures, appelées révolutions scientifiques, sont analogues à un renversement des représentations savantes.

2. Canguilhem fit une place à ce qu'il appelait l'idéologie scientifique : cette dernière était scientifique au sens où elle prenait modèle sur une théorie scientifique, mais elle était idéologique au sens où elle s'étendait au-delà du domaine d'applicabilité de cette théorie.

3. Le génotype est la totalité ou une partie donnée de la composition génétique (information génétique) d'un individu.

4. Le phénotype est la totalité des caractères observables d'un individu, à l'ensemble des échelles : macroscopique, cellulaire et moléculaire.

5. Les objectifs de ce programme européen sont :- constater comment sont enseignés des thèmes sensibles comme l'éducation à la santé, l'évolution, l'éducation à l'environnement...- analyser les 
interactions entre connaissances scientifiques et systèmes de valeurs. En fonction de quels paramètres ces connaissances et leur enseignement varient-ils? Du contexte socio-économique, culturel, religieux, de l'histoire récente du pays, de la discipline enseignée... ?

6. L'héréditarisme stipule que les phénotypes dont les comportements peuvent s'expliquer seulement par des déterminismes génétiques simples. Il est qualifié d'implicite lorsque il n'est pas déclaré mais qu'on peut l'identifier par l'utilisation de critères et d'indicateurs convenables.

\section{RÉSUMÉS}

Dans la communauté scientifique, l'ère du "tout-génétique" est remplacée par le paradigme de l'épigénétique qui met l'accent sur l'interaction entre génome et environnement. Existe-t-il dans les manuels scolaires des traces d'innéisme et éventuellement de sexisme ou de racisme associés à l'innéisme ? L'analyse comparative des chapitres qui traitent de la génétique humaine de cinq pays méditerranéens permet de dire qu'il y a une certaine survivance de conceptions innéistes dans ces manuels, plus ou moins marquée selon les pays, mais sans trace de représentations sexistes ou racistes. Les manuels peuvent être un moyen essentiel de promotion de valeurs citoyennes.

The present study aims to see up to which point national specificities are found in the chapters of the textbooks related to human genetics, among five countries around the Mediterranean Sea. Inside the scientific community, the era of "all-genetic" is now replaced by a new paradigm : the epigenetics, focused on interaction between the genes and their environment. The comparative analysis of textbooks shows a certain persistence of innatism in the five countries but in different ways. We never found a link between this innatism and some sexism or racism. The textbooks can be efficient to promote citizenship values.

\section{INDEX}

Mots-clés : manuel scolaire, valeurs, innéisme, sexisme

Keywords : biology textbooks, values, innatism, sexism

\section{AUTEURS}

\section{BOUJEMAA AGORRAM}

EREF, Ecole Normale Supérieure, Université Cadi Ayyad, Marrakech, Maroc

\section{SILVIA CARAVITA}

Istituto di Scienze e Tecnologie della Cognizione del C.N.R, Rome, Italie

\section{JÉRÉMY CASTÉRA}

EPS-LIRDHIST, Université Lyon 1, France 


\section{PIERRE CLÉMENT}

Université Lyon 1, France

\section{FÉRIDA KHAMMAR}

Université Sciences et Technologie Houari Boumediene, Alger, Algérie

SABAH SELMAOUI

EREF, École Normale Supérieure, Université Cadi Ayyad, Marrakech, Maroc? 